\title{
The latitudinal structure of the nightside outer magnetosphere of Saturn as revealed by velocity moments of thermal ions
}

\author{
Z. Nemeth ${ }^{1}$, K. Szego ${ }^{1}$, L. Foldy ${ }^{1}$, M. G. Kivelson ${ }^{2,3}$, X. Jia ${ }^{3}$, K. M. Ramer ${ }^{3}$, S. W. H. Cowley ${ }^{4}$, G. Provan ${ }^{4}$, and \\ M. Thomsen ${ }^{5}$ \\ ${ }^{1}$ Institute for Particle and Nuclear Physics, Wigner Research Centre for Physics, Budapest, Hungary \\ ${ }^{2}$ Atmospheric, Oceanic, and Space Sciences, University of Michigan, Ann Arbor, MI, USA \\ ${ }^{3}$ Earth and Space Sciences, University of California, Los Angeles, CA, USA \\ ${ }^{4}$ Department of Physics and Astronomy, University of Leicester, Leicester, UK \\ ${ }^{5}$ Planetary Science Institute, Tucson, AZ, USA \\ Correspondence to: Z. Nemeth (nemeth.zoltan@wigner.mta.hu)
}

Received: 3 November 2014 - Revised: 14 August 2015 - Accepted: 3 September 2015 - Published: 30 September 2015

\begin{abstract}
In this study we investigate the latitudinal behavior of the azimuthal plasma velocities in the outer magnetosphere of Saturn using the numerical ion moments derived from the measurements of the Cassini Plasma Spectrometer. One of the new results presented is that although these moments display some scatter, a significant positive correlation is found to exist between the azimuthal velocity and the plasma density, such that on average, the higher the density the higher the rotation speed. We also found that both the azimuthal velocity and the density anticorrelate with the magnitude of the radial component of the magnetic field and drop rapidly with increasing distance from the magnetic equator. The azimuthal velocities show periodic behavior with a period near the planetary rotation period, which can also be explained by the strong dependence on magnetic latitude, taking into account the flapping of the magnetodisk. It is thus found that the dense plasma near the magnetic equator rotates around the planet at high speed, while the dilute plasma at higher latitudes in the northern and southern hemispheres rotates significantly slower. The latitudinal gradient observed in the azimuthal speed is suggested to be a direct consequence of the sub-corotation of the plasma in the outer magnetosphere, with highest speeds occurring on field lines at lowest latitudes mapping to the rapidly rotating inner regions of the plasma sheet, and the speed falling as one approaches the lobe, where the field lines are connected to strongly subcorotating plasma.
\end{abstract}

Keywords. Magnetospheric physics (magnetospheric configuration and dynamics; planetary magnetospheres; plasma sheet)

\section{Introduction}

The first in situ measurements in the magnetosphere of Saturn were obtained by the Pioneer 11 and the Voyager 1 and 2 spacecraft, in 1979, 1980, and 1981, respectively. After the short visits of these probes in the Kronian system many open questions remained because these short encounters had a rather limited spatial and temporal coverage of the magnetosphere. In 2004 the Cassini orbiter arrived at Saturn and began its comprehensive observations of the Kronian magnetosphere. The results of the first few years of the Cassini era have been reviewed by several review papers (Gombosi et al., 2009; Mitchell et al., 2009; Mauk et al., 2009). Through the new findings the structure of the Kronian magnetosphere began to unfold.

Since Saturn is a fast rotator, centrifugal effects play a very important role in its rotating magnetosphere. The interplay of the centrifugal force acting on the charged particles, the magnetic stress, and the kinetic pressure of the particles leads to the formation of a complex plasma/magnetic field structure called the magnetodisk (Arridge et al., 2006; Achilleos et al., 2010). Most of the plasma is concentrated in this rotating disk, which is situated near the equatorial plane and perturbs (stretches) the planetary magnetic field. The shape 
of the magnetodisk is also affected by the solar wind. Before equinox, the angle of attack of the solar wind caused the magnetodisk to acquire a bowl shape (Arridge et al., 2008). In addition to this static deformation a vertical flapping motion relative to an outside observer was also detected, its period being close to the planetary rotation period.

In the Kronian magnetosphere many properties of the observed fields, particles and waves show periodic variations, associated with the planetary rotation (Arridge et al., 2011a; Provan et al., 2012). The periodicity observed in Saturn kilometric radiation (SKR) is a good example of these periods (Gurnett et al., 2005). Periodic variations were also found in the magnetic field (Espinosa and Dougherty, 2000; Andrews et al., 2008, 2012; Provan et al., 2012). Burch et al. (2009) have established that the SKR period also organizes the heavy ion data sampled by the Cassini Plasma Spectrometer (CAPS). The electron density in the vicinity of Titan's orbital distance was also found to be periodic (Arridge et al., 2008), the density modulation being explained by the periodic motion of the plasma sheet.

Most of the results of the primary and early equinox mission pointed toward a relatively simple picture of the magnetodisk: a rotating asymmetric bowl-shaped current sheet, exhibiting apparent vertical flapping motion. Arridge et al. (2011a) proposed a "simple structural model" to describe the shape and the flapping of the magnetodisk.

Since the equinox several new discoveries modified this simple picture into a more complex and less clear image. Gurnett et al. (2009) found that the periodicity in the SKR emission differs in the northern and southern hemispheres and both periodicities vary slowly. Andrews et al. (2012) and Provan et al. (2012, 2013) investigated magnetic field modulations in Saturn's magnetosphere. They have shown that in the high-latitude regions of the northern and southern hemispheres single period modulations can be observed, but near the current sheet dual periodicities are characteristic. It was shown that the interplay of the two periodicities in the equatorial region can cause phase shifts and slow modulation of the amplitude of the periodic variations (beats). It is also suggested that the thickness of the plasma sheet varies with the beat period. These new results motivated Szego et al. (2013) to modify the simple structural model of Arridge et al. (2011a). In the new model the dual periodicities of the equatorial region are also taken into account.

Periodic variations were found in the density of thermal ions as well (Nemeth et al., 2011; Szego et al., 2011, 2012, 2013; Arridge et al., 2011b). The thermal plasma sheet properties are shown to be different for different ion species. The proton sheet is smoothly modulated by the flapping of the magnetodisk; in the nightside the heavy ions form a narrow sheet surrounding the magnetic equator, and when the nominal center of the plasma sheet is crossed $\left(B_{\mathrm{r}}=0\right)$, highdensity heavy ion peaks can be observed. The periodicity of these heavy rich events was found to be close to the southern SKR period. Morooka et al. (2009) have shown that quasi- periodic variations of the electron density can be observed all over the magnetosphere, even in the lobe region beyond $L=15$. They also found that Saturn's magnetosphere has a strong longitudinal asymmetry.

Various models have been suggested to explain the properties of the Kronian magnetosphere (Espinosa et al., 2003; Southwood and Kivelson, 2007; Goldreich and Farmer, 2007; Gurnett et al., 2007; Southwood and Kivelson, 2009; Khurana et al., 2009; Carbary and Mitchell, 2013). Recently Jia and Kivelson (2012) proposed a numerical model, which agrees well with most of the experimental results currently known. The model assumes an ionospheric source of rotational asymmetry. There are twin-vortical structures in the southern and northern ionosphere in the model, rotating at the southern and northern SKR period respectively. Using an Ionosphere Electrodynamics (IE) solver together with the global three-dimensional magnetohydrodynamic (MHD) model they computed the effects of these ionospheric vortices on the magnetosphere. The results show the flapping and breathing magnetodisk, as well as the dual periodicities in magnetic and plasma data. Jia and Kivelson (2012) predict variations of the plasma velocities. The velocity components show periodic modulation, the azimuthal speed $V_{\varphi}$ varying approximately in phase with the density. Here we show that this model result is supported by data.

Several papers studied the velocity variations of the plasma in the Kronian magnetosphere. Müller et al. (2010) studied the azimuthal plasma velocity in the inner magnetosphere of Saturn. Ramer et al. (2012) investigated velocity periodicities in the inner magnetosphere near the equatorial plane. Wilson et al. (2013) found evidence of a global electric field in the inner magnetosphere from radial velocity measurements. McAndrews et al. (2009) presented a bulk ion flow map from the nightside equatorial region of Saturn's magnetosphere. Thomsen et al. (2014) provided a radial profile of the nightside azimuthal velocity. Carbary and Mitchell (2014) used energetic neutral atom (ENA) images to determine the rotational speeds of the plasma by measuring the speed of features ("blobs") appearing in the images; their results show good agreement with in situ plasma measurements.

These papers established the basic behavior of the azimuthal flow with respect to radial distance near the equatorial plane in the nightside magnetosphere of Saturn, falling from near-rigid corotation at a distance of $\sim 3$ Saturn radii $\left(R_{\mathrm{S}}\right)$, to $\sim 50 \%$ of rigid corotation or less at $\sim 20 R_{\mathrm{S}}$ and beyond. We extend these studies to include higher latitude passes in the outer magnetosphere to the orbit of Titan and beyond. The broad latitudinal coverage of these passes reveals the latitudinal structure of the flows in the outer magnetosphere together with a quasi-periodic variation associated with this structure. We investigate the azimuthal flow velocities and their periodicities using numerical ion moments (Thomsen et al., 2010) derived from the measurements of the Cassini Plasma Spectrometer (CAPS) onboard Cassini 
(Young et al., 2004). Our analysis covers data from the beginning of 2005 to the end of 2009 , from southern summer to equinox conditions. As the spacecraft spent a significant amount of time on the nightside only in years 2006 and 2009, our analysis is based on the data of these 2 years. This long data acquisition period gives us excellent statistics, which is essential for the investigation of the structure of the magnetodisk.

\section{Data analysis}

We started our investigations with several case studies. In 200911 consecutive orbits of the Cassini spacecraft (from Rev 108 to Rev 119, with the exception of Rev 118) were such that each of them intersected the equatorial plane of Saturn at about 22:00 LT, and approached the equator there with decreasing inclination. Along all of these orbits Cassini flew by Titan, starting with T52 (day 94 of 2009 (4 April) 01:47:48), and finishing with T62 (day 285 of 2009 (12 October) 08:36:25). The Saturn equinox was on 11 August 2009, so this set of orbits is of particular interest for studying the nightside magnetodisk of Saturn under near-equinoctial conditions.

For each of these orbits we examined the radial component of the magnetic field $\left(B_{\mathrm{r}}\right)$, together with the azimuthal velocity and the density of the proton species derived by Thomsen et al. (2010). Figure 1 shows two of these plots, for the T57 and T59 Titan encounters respectively. The plasma data are missing, where the moments calculation could not give a reliable result. This can happen due to unfavorable spacecraft conditions (CAPS is not actuating or the spacecraft is rolling; rolling is a problem for moment analysis and it is required that during a measurement cycle the particles hitting different anodes should not mix), due to low signal-to-noise ratio, or where the numerical method does not converge. Most of the data gaps in the figure reside where the plasma density is presumably very low, which leads to low signal-to-noise ratio. In Fig. 1 the ion plasma data for which the direction of the ion flow was inside the field of view of the CAPS instrument are distinguished from other reliable data by color; the lines are green for "corotation in field of view" and blue for the other reliable velocity and density moments. We removed the data from the immediate vicinity of Titan, since the magnetic field changes due to Titan's induced magnetosphere, and the ion peaks due to dense ionospheric plasma are of no or little consequence regarding the large-scale variations of the Kronian magnetosphere.

The data are heavily loaded with fluctuations due to changing physical environment, but the presence of variations with periodicity near the planetary rotation period is evident. By periodicity here we mean recurrent peaks generally occurring in every planetary rotation period in the vicinity of the magnetic equator. An almost one to one correspondence can be observed between the periodically recurring velocity and density peaks. (See Fig. 1. We have drawn a few vertical lines to help visualize alignment.) It should be noted that these peaks also correspond to dips or zero-crossings in the radial magnetic field. The recurring peaks are present along the other orbit segments as well; almost all of the peaks coincide with the periodic magnetic signatures, which can be identified as those times when the spacecraft approaches or crosses the wavy central sheet of the magnetodisk. The periodic behavior of the magnetic field has been established by using a robust analysis technique (see e.g. Provan et al., 2012). Although our sporadic data set does not allow for the usage of that technique, the observation that velocity and magnetic field signatures coincide strongly suggests that the two phenomena have similar periodicities.

The numerical simulations of Jia and Kivelson (2012) found a direct correlation between the azimuthal velocity and the density. Our case studies show one to one correspondence between these two quantities. Empirically based theoretical models of the density e.g. Persoon et al. (2009) show that the density depends strongly on the distance from the central sheet of the magnetodisk, the density being highest in the central sheet and steadily decreasing towards the lobes. This dependence together with the vertical flapping motion of the magnetodisk leads to the periodic modulation of the measured density along the orbit of the spacecraft, which was recently found in Cassini ion data (Nemeth et al., 2011; Szego et al., 2011, 2013). This suggests that the observed periodic modulation of the azimuthal velocity also correlates with the motion of the magnetodisk. Near the crossings the velocity is high, while the plasma is slower farther away.

Due to the strong fluctuations and the sporadic nature of the data, the case studies are not entirely sufficient to answer every question. It is important to verify whether the velocity variations we see are really due to the variation of the distance between the spacecraft and the central sheet of the magnetodisk. How does this relationship develop from the plasma sheet to the lobes? How does the velocity variation correlate with other parameters of the plasma, e.g. the density and the radial magnetic field? What are their quantitative relationships? What are the implications concerning the structure of the magnetosphere?

To examine the correlation suggested by the models and our case studies, and also to support our findings about the periodic velocity variations, we created velocity-density scatter plots using data not only from the orbit segments of the case studies, but all the available and reliable moments data obtained during 2009 and 2006, the 2 years when the Cassini explored the nightside outer magnetosphere. Our particular interest is the nightside outer magnetosphere, so only data are employed for which the Kronian local time (LT) is less than 3 hours from midnight, and the distance from Saturn is between 15 and $28 R_{\mathrm{S}}$ (the radial range where Cassini explored our region of interest). The upper left panel of Fig. 2 (for 2009) shows these scatter plots for protons; the upper right panel of Fig. 2 shows similar data for heavy ions. Both 

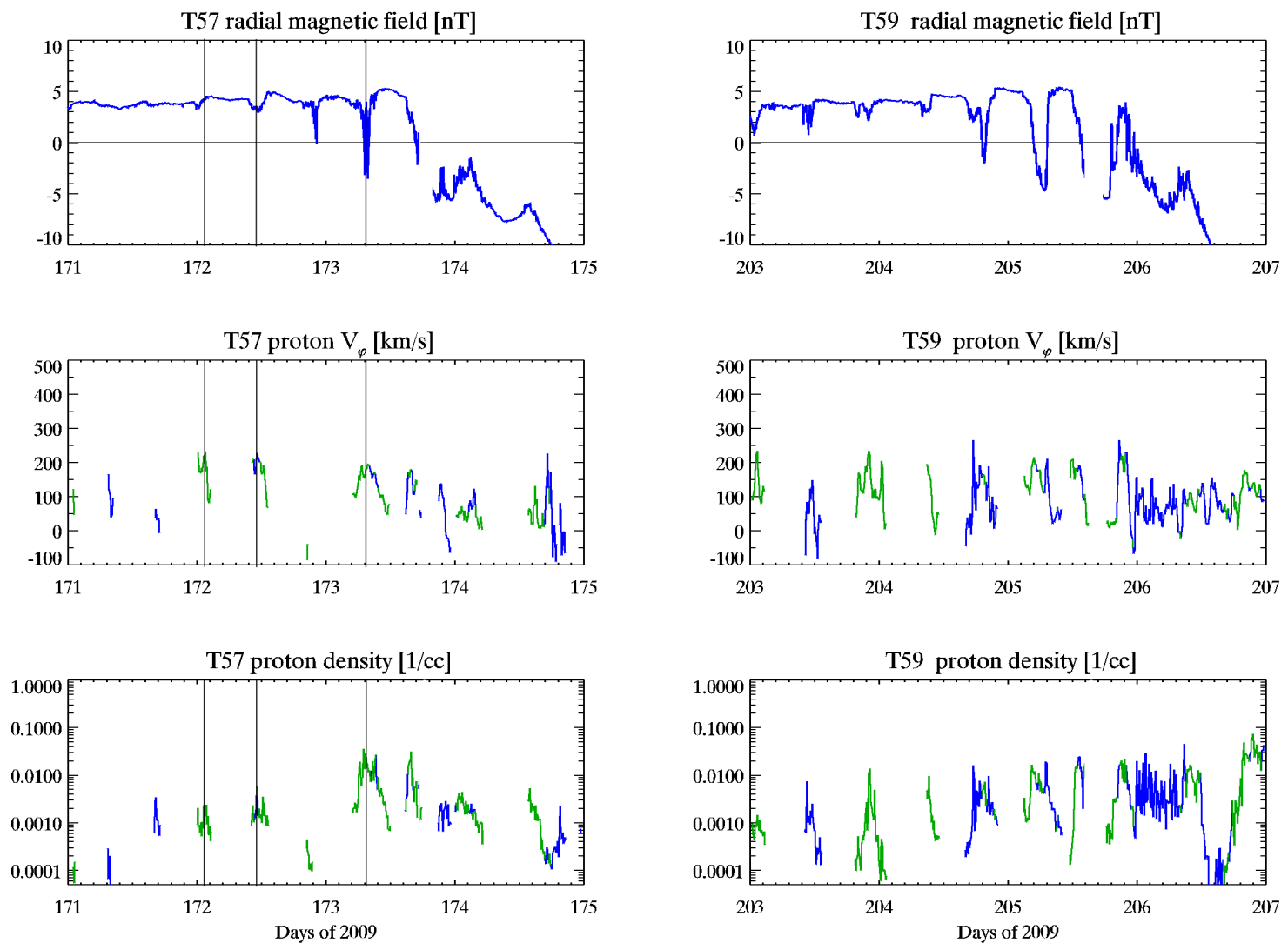

Figure 1. Radial magnetic field (top panel), azimuthal velocity (middle panel) and proton density (bottom panel) for two 4-day long orbit segments containing the T57 (on the left) and T59 (on the right) Titan encounters.

plots show a direct correlation between the azimuthal velocity and the density for both light and heavy ions. The Pearson correlation coefficient is 0.49 for protons, 0.54 for heavy ions. This means moderate or moderately strong correlation. Due to the high number of the samples (thousands of measurement points) the significance of this result is very high, far over the five sigma level. (The significance of the correlation indicates whether a correlation proves the relationship of the two quantities or not. The measure of significance is the p-value, which tells how unlikely it is that a correlation coefficient will occur without relationship in the population. For a given value of the correlation coefficient, the larger the sample size, the smaller the probability of this coefficient occurring by chance. A coefficient around 0.5 for thousands of points means very high significance.) This result proves beyond doubt that there is a relationship between the density and the azimuthal velocity, they increase and decrease together. The result is even more remarkable if we consider that the correlation is found on a large, year-long sample, in which other effects of the very dynamic Kronian magnetosphere cause a strong scatter. The steeper curve for the heavy ions arises because their number density depends strongly on the distance from the central sheet (e.g. Persoon et al., 2009). As we move away from the magnetic equator, the heavy den- sity decreases much more rapidly than the proton density, but the speeds of the two plasma species are comparable. Thus the same velocity variation corresponds to a much stronger density variation for the heavy ions. The bottom panels of Fig. 2 show box-plots for the same data set. The horizontal lines of each box represent the first, second and third quartiles of the corresponding bin. We have chosen the bins so that there are the same number of points in each bin, this is why the width of the bins varies. We have discarded the first and last bin of each plot, because those were much wider than the others indicating very high velocity scatter. The straight red lines are linear fits $(\log (\varrho)=a v+b)$ for the mean values of the bins, where $a=0.006$ and $b=-2.95$ for protons; and $a=0.015$ and $b=-4.0$ for heavy ions. As for the proton data of year 2009 the linear behavior clearly breaks down for very high and low speeds, the fit for that data set only contains the seven linear bins. In summary, near the magnetic equator in the central region of the plasma sheet, where the density is high, we usually find high azimuthal speeds as well. In the lobes the azimuthal velocity is much lower. The velocity changes $100-150 \mathrm{~km} \mathrm{~s}^{-1}$ between the two regions, while at the same time the density changes 0.6 and roughly 2 orders of magnitude for protons and heavy ions respectively. In this the observations seem to be in agreement with the 

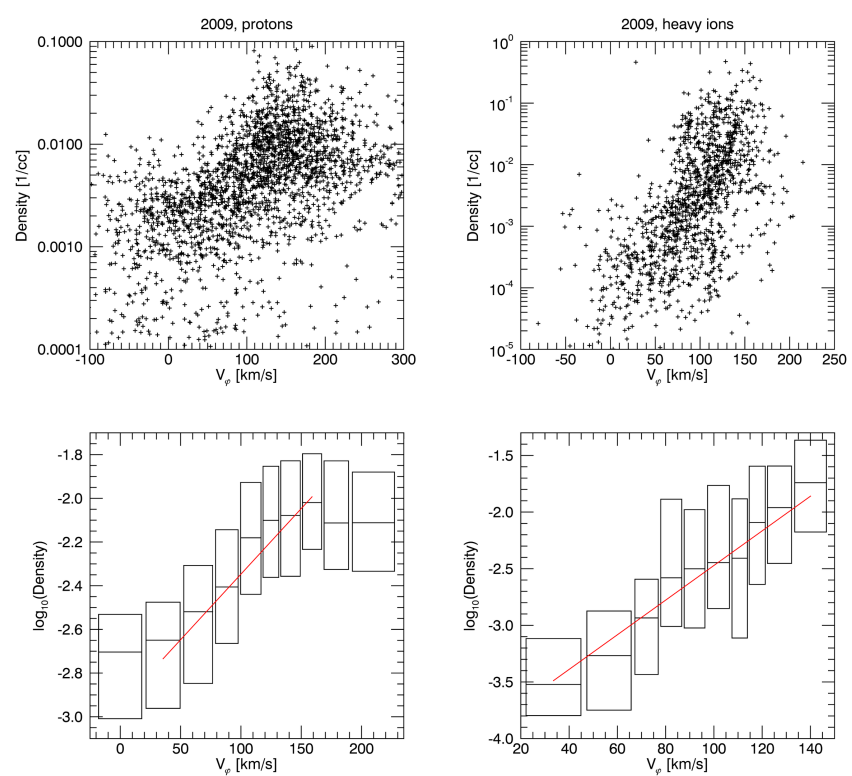

Figure 2. Azimuthal velocity - density relationship. The left panels show proton data, the right panels show water-group data, measured in the nightside outer magnetosphere of Saturn in year 2009. Upper panels: scatter plots, bottom panels: box-plots. The three horizontal lines of each box represent the first, second and third quartiles of the corresponding bin. There is an equal number of points in each bin, this is why the width of the bins vary. The red line is a linear fit for the mean values of the bins it crosses.

basic assumptions about the nature of the flow in the models of Cowley et al. (2004) and Cowley et al. (2008), where strongly sub-corotating flow was found in the polar regions of open field lines, and near corotation was detected in the equatorial region. Sometimes we even see negative values in the lobes, but it seems there is rather strong scatter around zero $\mathrm{km} \mathrm{s}^{-1}$ in the dilute plasma at high latitudes.

Assuming that the velocity peaks on the central sheet of the magnetodisk, and the $v(z)$ function is smooth (where $z$ is the distance from the central sheet of the magnetodisk), we can approximate $v(z)$ in lowest order as $v(z)=V(1-$ $\left.z^{2} / Z^{2}\right)$. Here $V$ is the peak velocity and $Z$ is the length scale characteristic of the falloff of the velocity. If we can approximate the density as $\varrho(z)=\varrho_{0} \exp \left(-z^{2} / \lambda^{2}\right)$, where $\varrho_{0}$ is the peak value and $\lambda$ is the scale height of the density, then the $\varrho(v)$ function can be written as $\log \left(\varrho(v) / \varrho_{0}\right)=$ $Z^{2} / \lambda^{2}(v / V-1)$. Thus from the fitting parameters we can calculate the peak velocity, together with the ratio of the two length scales: $Z^{2} / \lambda^{2}=-b+\log \left(\varrho_{0}\right), V=-\left(b-\log \left(\varrho_{0}\right)\right) / a$.

It is obvious from Fig. 2 that the straight lines are not an ideal fit for the data. Hence the parameters calculated from the linear fits should not be used to determine the relation among physical quantities, but still, they can be useful in evaluating the seasonal variations, or the differences in the behavior of the two ion species. In Fig. 3 plots similar to those of Fig. 2 are shown for 2006. The orbit segments in
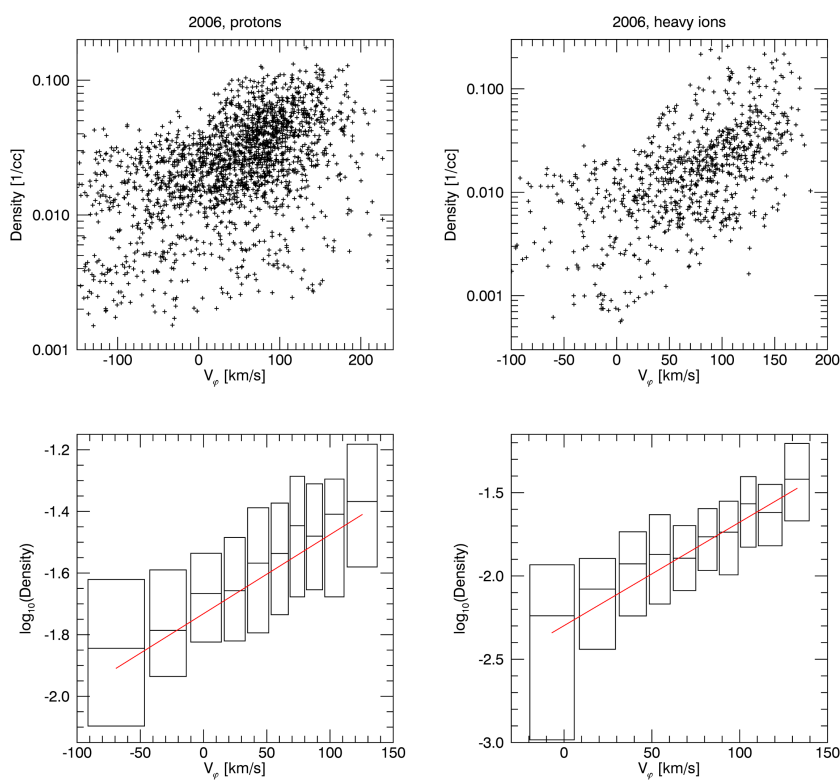

Figure 3. The same as in Fig. 2 but for year 2006.

the nightside magnetosphere are very similar for 2006 and 2009, although the 2009 passes contain a few fast latitude scans. Only in these 2 years flew Cassini in the nightside. These two sets of orbits provide the best opportunity to study the long-term stability or variations of the observed relationships. We use the same radial and local time ranges as before. The correlations between density and azimuthal velocity are definitely present in 2006 as well (correlation coefficients are 0.41 and 0.49 for protons and heavy ions respectively). The densities seem to be somewhat higher in this year, with less variation. The azimuthal velocity is somewhat lower (at least for the protons); the velocity-density correlation scatter plots show a more gradual incline. We do not know the cause of these long-term variations, but it is possible that seasonal effects influence the density and velocity distributions in the nightside outer magnetosphere.

Comparing the quantitative results derived from the fitting parameters, we find that in 2009 the ratio of the velocity and density scale height for the heavy ions is 2.4 times the ratio for the protons where the different scale heights reflect the stronger confinement of the heavy component. The corresponding value is 2 for 2006. If we compare the ratio of the velocity and density scale height of a single species but for different years, we find that the confinement is much stronger in 2009. The ratio of the 2009 and 2006 data for protons is 2.3 ; it is 2.6 for heavy ions. This means that in the nightside outer magnetosphere the thickness of the plasma sheet in 2009 is less than half the 2006 value. This explains the changes in the peak density as well - the plasma is concentrated around the magnetic equator, so its density has a higher peak value, but it falls off faster with height above the magnetic equator. The peak velocities also show significant 
seasonal variation. For 2006 the fits give $110-130 \mathrm{~km} \mathrm{~s}^{-1}$ for this region, roughly the same for the two species. For 2009 the values are much higher, $120-150$ for heavy ions and 160180 for protons.

Since by definition $B_{\mathrm{r}}$ is zero at the magnetic equator, and in this vicinity varies near-linearly with distance from the central sheet at a given radial distance, this quantity is a good organizational parameter for study of the structure of the magnetodisk near the central sheet. The dependence on the distance from the central sheet is even clearer, if we employ the residual radial magnetic field $\left(B_{\mathrm{r}}-B_{\mathrm{rd}}\right.$ where $B_{\mathrm{rd}}$ is the radial component of the dipole field), since the radial dependence of the dipole field, which can cause significant variations along an arbitrary path, is removed. The radial field is known to be periodic (e.g. Andrews et al., 2012; Provan et al., 2012), so the $B_{\mathrm{r}}$ dependence of the velocity can provide a further insight into the periodic behavior of the velocity variations as well.

The top panels of Fig. 4 show azimuthal velocity versus residual radial magnetic field scatter plots for 2 years (2006 and 2009) for the light ion component. These 2 years provide the best opportunity to study the nightside outer magnetosphere. The points of the left panel represent data from 2009, the right panel contains data from 2006. As the radial dependence of the magnetic field could blur the picture, for the purpose of this analysis we constrained our radial range inside the $4.5 R_{\mathrm{S}}$ vicinity of the intersection of the orbit segments. The boxes of the lower panels represent the lower quartile, median and upper quartile of the data for $1 \mathrm{nT}$ wide magnetic field intervals. The data show the same trend for both years, namely near the magnetic equator we find high azimuthal speeds, while moving away from the magnetic equator we encounter slower and slower plasma, and in the lobes the rotation of the plasma is very slow. The medians of the boxplots indicate that the azimuthal velocity in the lobes is nearly zero, while the central sheet is rotating with approximately $160 \mathrm{~km} \mathrm{~s}^{-1}$ in 2009; the same value for 2006 is $90 \mathrm{~km} \mathrm{~s}^{-1}$.

Our results suggest that the Kronian magnetodisk has a dense, rapidly rotating central plasma sheet with the rotation rate low in the lobes, and gradual variation in between. To explain this behavior we propose a simple model, which we call the "rigid L-shell model". In this model each L-shell rotates independently from the other L-shells, with individual shells rotating approximately as a rigid body, with constant angular velocity along each field line. Since a shell's rotation speed is determined by its dense equatorial region, which rotates slower farther away from the planet (sub-corotation), a latitude scan intersects slower and slower shells as it moves away from the magnetic equator.

This model readily explains the observed periodicity as well if we take into account the flapping of the magnetodisk. Due to this flapping motion, the spacecraft is periodically immersed in the dense fast-moving plasma of the central plasma sheet, which causes the periodic occurrence of high-speed "streams".
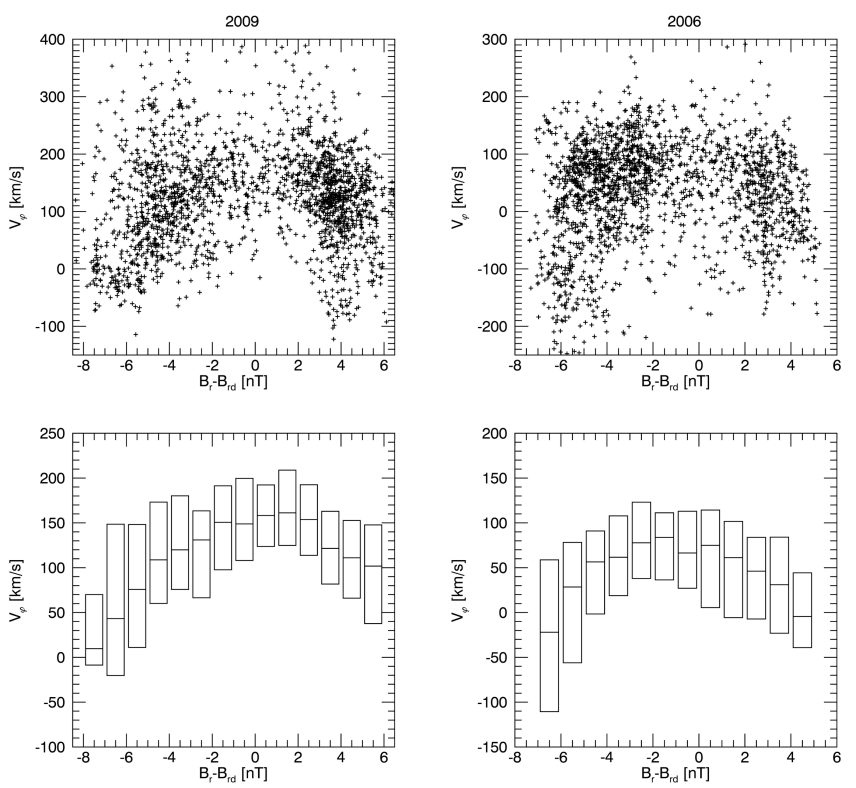

Figure 4. Residual magnetic field - azimuthal velocity scatter plots (upper panels) and box-plots (lower panels) for proton data measured in the nightside outer magnetosphere of Saturn. Left panels: 2009, right: 2006. The three horizontal lines of each box represent the first, second and third quartiles of the corresponding bin. The velocity approaches zero far away from the magnetic equator, while the central sheet of the magnetodisk rotates rapidly. (Its speed is approximately $160 \mathrm{~km} \mathrm{~s}^{-1}$ for 2009 and $90 \mathrm{~km} \mathrm{~s}^{-1}$ for 2006.)

\section{Conclusions}

We investigated the modulation of thermal ion plasma velocities in the nightside outer magnetosphere of Saturn. Since we were interested in the 3-D structure of the magnetosphere we also included high latitude data gathered along high inclination orbits.

- We observed a significant positive correlation between $V_{\varphi}$ and the density, such that on average, the higher the density the higher the rotation speed. This agrees well with the numerical model of Jia and Kivelson (2012).

- Both $V_{\varphi}$ and the density anticorrelate with the absolute value of the radial component of the magnetic field $\left(B_{\mathrm{r}}\right)$.

- We used $B_{\mathrm{r}}$ as an indicator of the distance from the central sheet of the magnetodisk, and found that the dense plasma near the magnetic equator rotates around the planet at high speed, but the dilute plasma of the higher latitudes of the northern and southern hemispheres is rotating significantly slower. The azimuthal velocity in the lobes is nearly zero, while the central sheet is rotating with roughly $160 \mathrm{~km} \mathrm{~s}^{-1}$ in 2009 ; the same value for 2006 is $90 \mathrm{~km} \mathrm{~s}^{-1}$.

- We found that the ion velocities exhibit periodic modulation. The period of the modulation is near the ro- 
tational period generally observed by magnetic and plasma measurements (Provan et al., 2012; Andrews et al., 2012; Szego et al., 2011, 2013). The observed periodicity is compatible with the magnetic phases of Provan et al. (2011) however the data coverage is not good enough to allow a stronger statement.

These findings can be explained by supposing that each Lshell rotates at fixed angular velocity but this velocity varies from shell to shell. Thus the latitudinal gradient observed in the azimuthal speed is a direct consequence of the subcorotation of the plasma in the outer magnetosphere, with highest speeds occurring on those field lines at lowest latitudes mapping to the rapidly rotating inner regions of the dense plasma sheet, and the speed falling as one approaches the lobe, where the field lines are connected to far away strongly sub-corotating plasma. Thus one can explore the whole radial range of equatorial velocities at a fixed distance by examining the velocity as a function of flux shell - parameterized by residual $B_{\mathrm{r}}$ ).

Acknowledgements. The work of Z. Nemeth, K. Szego, and L. Foldy was supported by the Hungarian National Development Agency (NFÜ) under contract URKUT_1-2011-0011. SWHC and GP were supported by STFC grant ST/K001000/1. Work at PSI was supported by the NASA Cassini program through JPL contract 1243218 with Southwest Research Institute. In the US the Cassini project is managed by the Jet Propulsion Laboratory for NASA. The authors also thank M. Dougherty and the MAG team for providing the magnetic field data. Cassini MAG data and Cassini ion moments are available at the Planetary Data System (https: //pds.jpl.nasa.gov/).

The topical editor C. Owen thanks the two anonymous referees for help in evaluating this paper.

\section{References}

Achilleos, N., Guio, P., and Arridge, C. S.: A model of force balance in Saturn's magnetodisc, Mon. Not. R. Astron. Soc., 401, 23492371, doi:10.1111/j.1365-2966.2009.15865.x, 2010.

Andrews, D. J., Bunce, E. J., Cowley, S. W. H., Dougherty, M. K., Provan, G., and Southwood, D. J.: Planetary period oscillations in Saturn's magnetosphere: Phase relation of equatorial magnetic field oscillations and Saturn kilometric radiation modulation, J. Geophys. Res., 113, 9205, doi:10.1029/2007JA012937, 2008.

Andrews, D. J., Cowley, S. W. H., Dougherty, M. K., Lamy, L., Provan, G., and Southwood, D. J.: Planetary period oscillations in Saturn's magnetosphere: Evolution of magnetic oscillation properties from southern summer to post-equinox, J. Geophys. Res., 117, 4224, doi:10.1029/2011JA017444, 2012.

Arridge, C. S., Achilleos, N., Dougherty, M. K., Khurana, K. K., and Russell, C. T.: Modeling the size and shape of Saturn's magnetopause with variable dynamic pressure, J. Geophys. Res., 111, 11227, doi:10.1029/2005JA011574, 2006.

Arridge, C. S., Khurana, K. K., Russell, C. T., Southwood, D. J., Achilleos, N., Dougherty, M. K., Coates, A. J., and Leinweber, H. K.: Warping of Saturn's magnetospheric and magnetotail current sheets, J. Geophys. Res., 113, 8217, doi:10.1029/2007JA012963, 2008.

Arridge, C. S., André, N., Bertucci, C. L., Garnier, P., Jackman, C. M., Németh, Z., Rymer, A. M., Sergis, N., Szego, K., Coates, A. J., and Crary, F. J.: Upstream of Saturn and Titan, Space Sci. Rev., 162, 25-83, doi:10.1007/s11214-011-9849-x, 2011a.

Arridge, C. S., André, N., Khurana, K. K., Russell, C. T., Cowley, S. W. H., Provan, G., Andrews, D. J., Jackman, C. M., Coates, A. J., Sittler, E. C., Dougherty, M. K., and Young, D. T.: Periodic motion of Saturn's nightside plasma sheet, J. Geophys. Res., 116, 11205, doi:10.1029/2011JA016827, 2011b.

Burch, J. L., DeJong, A. D., Goldstein, J., and Young, D. T.: Periodicity in Saturn's magnetosphere: Plasma cam, Geophys. Res. Lett., 36, 14203, doi:10.1029/2009GL039043, 2009.

Carbary, J. F. and Mitchell, D. G.: Periodicities in Saturn's magnetosphere, Rev. Geophys., 51, 1-30, doi:10.1002/rog.20006, 2013.

Carbary, J. F. and Mitchell, D. G.: Keogram analysis of ENA images at Saturn, J. Geophys. Res., 119, 1771-1780, doi:10.1002/2014JA019784, 2014.

Cowley, S. W. H., Bunce, E. J., and O'Rourke, J. M.: A simple quantitative model of plasma flows and currents in Saturn's polar ionosphere, J. Geophys. Res., 109, 5212, doi:10.1029/2003JA010375, 2004.

Cowley, S. W. H., Arridge, C. S., Bunce, E. J., Clarke, J. T., Coates, A. J., Dougherty, M. K., Gérard, J.-C., Grodent, D., Nichols, J. D., and Talboys, D. L.: Auroral current systems in Saturn's magnetosphere: comparison of theoretical models with Cassini and HST observations, Ann. Geophys., 26, 2613-2630, doi:10.5194/angeo-26-2613-2008, 2008.

Espinosa, S. A. and Dougherty, M. K.: Periodic perturbations in Saturn's magnetic field, Geophys. Res. Lett., 27, 2785-2788, 2000.

Espinosa, S. A., Southwood, D. J., and Dougherty, M. K.: How can Saturn impose its rotation period in a noncorotating magnetosphere?, J. Geophys. Res.-Space, 108, 1086 , doi:10.1029/2001JA005084, 2003.

Goldreich, P. and Farmer, A. J.: Spontaneous axisymmetry breaking of the external magnetic field at Saturn, J. Geophys. Res., 112, 5225, doi:10.1029/2006JA012163, 2007.

Gombosi, T. I., Armstrong, T. P., Arridge, C. S., Khurana, K. K., Krimigis, S. M., Krupp, N., Persoon, A. M., and Thomsen, M. F.: Saturn's magnetospheric configuration, in: Saturn from CassiniHuygens, edited by: Dougherty, M. K., Esposito, L. W., and Krimigis, S. M., 203-255, Springer Netherlands, 2009.

Gurnett, D. A., Kurth, W. S., Hospodarsky, G. B., Persoon, A. M., Averkamp, T. F., Cecconi, B., Lecacheux, A., Zarka, P., Canu, P., Cornilleau-Wehrlin, N., Galopeau, P., Roux, A., Harvey, C., Louarn, P., Bostrom, R., Gustafsson, G., Wahlund, J.-E., Desch, M. D., Farrell, W. M., Kaiser, M. L., Goetz, K., Kellogg, P. J., Fischer, G., Ladreiter, H.-P., Rucker, H., Alleyne, H., and Pedersen, A.: Radio and Plasma Wave Observations at Saturn from Cassini's Approach and First Orbit, Science, 307, 1255-1259, doi:10.1126/science.1105356, 2005.

Gurnett, D. A., Persoon, A. M., Kurth, W. S., Groene, J. B., Averkamp, T. F., Dougherty, M. K., and Southwood, D. J.: The Variable Rotation Period of the Inner Region of Saturn's Plasma Disk, Science, 316, 442-445, doi:10.1126/science.1138562, 2007.

Gurnett, D. A., Lecacheux, A., Kurth, W. S., Persoon, A. M., Groene, J. B., Lamy, L., Zarka, P., and Carbary, J. F.: Discov- 
ery of a north-south asymmetry in Saturn's radio rotation period, Geophys. Res. Lett., 36, 16102, doi:10.1029/2009GL039621, 2009.

Jia, X. and Kivelson, M. G.: Driving Saturn's magnetospheric periodicities from the upper atmosphere/ionosphere: Magnetotail response to dual sources, J. Geophys. Res., 117, 11219, doi:10.1029/2012JA018183, 2012.

Khurana, K. K., Mitchell, D. G., Arridge, C. S., Dougherty, M. K., Russell, C. T., Paranicas, C., Krupp, N., and Coates, A. J.: Sources of rotational signals in Saturn's magnetosphere, J. Geophys. Res., 114, 2211, doi:10.1029/2008JA013312, 2009.

Mauk, B. H., Hamilton, D. C., Hill, T. W., Hospodarsky, G. B., Johnson, R. E., Paranicas, C., Roussos, E., Russell, C. T., Shemansky, D. E., Sittler, E. C., and Thorne, R. M.: Fundamental Plasma Processes in Saturn's Magnetosphere, in: Saturn from Cassini-Huygens, edited by: Dougherty, M. K., Esposito, L. W., and Krimigis, S. M., 281-331, Springer Netherlands, 2009.

McAndrews, H. J., Thomsen, M. F., Arridge, C. S., Jackman, C. M., Wilson, R. J., Henderson, M. G., Tokar, R. L., Khurana, K. K., Sittler, E. C., Coates, A. J., and Dougherty, M. K.: Plasma in Saturn's nightside magnetosphere and the implications for global circulation, Planet. Space Sci., 57, 1714-1722, doi:10.1016/j.pss.2009.03.003, 2009.

Mitchell, D. G., Carbary, J. F., Cowley, S. W. H., Hill, T. W., and Zarka, P.: The Dynamics of Saturn's Magnetosphere, in: Saturn from Cassini-Huygen-, edited by: Dougherty, M. K., Esposito, L. W., and Krimigis, S. M., 257-279, Springer Netherlands, 2009.

Morooka, M. W., Modolo, R., Wahlund, J.-E., André, M., Eriksson, A. I., Persoon, A. M., Gurnett, D. A., Kurth, W. S., Coates, A. J., Lewis, G. R., Khurana, K. K., and Dougherty, M.: The electron density of Saturn's magnetosphere, Ann. Geophys., 27, 29712991, doi:10.5194/angeo-27-2971-2009, 2009.

Müller, A. L., Saur, J., Krupp, N., Roussos, E., Mauk, B. H., Rymer, A. M., Mitchell, D. G., and Krimigis, S. M.: Azimuthal plasma flow in the Kronian magnetosphere, J. Geophys. Res., 115, 8203, doi:10.1029/2009JA015122, 2010.

Nemeth, Z., Szego, K., Bebesi, Z., Erdos, G., Foldy, L., Rymer, A., Sittler, E. C., Coates, A. J., and Wellbrock, A.: Ion distributions of different Kronian plasma regions, J. Geophys. Res., 116, 9212, doi:10.1029/2011JA016585, 2011.

Persoon, A. M., Gurnett, D. A., Santolik, O., Kurth, W. S., Faden, J. B., Groene, J. B., Lewis, G. R., Coates, A. J., Wilson, R. J., Tokar, R. L., Wahlund, J.-E., and Moncuquet, M.: A diffusive equilibrium model for the plasma density in Saturn's magnetosphere, J. Geophys. Res., 114, 4211, doi:10.1029/2008JA013912, 2009.

Provan, G., Andrews, D. J., Arridge, C. S., Coates, A. J., Cowley, S. W. H., Cox, G., Dougherty, M. K., and Jackman, C. M.: Dual periodicities in planetary-period magnetic field oscillations in Saturn's tail, J. Geophys. Res., 117, 1209, doi:10.1029/2011JA017104, 2012.

Provan, G., Andrews, D. J., Arridge, C. S., Coates, A. J., Cowley, S. W. H., Milan, S. E., Dougherty, M. K., and Wright, D. M.: Polarization and phase of planetary-period magnetic field oscillations on high-latitude field lines in Saturn's magnetosphere, J. Geophys. Res., 114, 2225, doi:10.1029/2008JA013782, 2009.

Provan, G., Cowley, S. W. H., Sandhu, J., Andrews, D. J., and Dougherty, M. K.: Planetary period magnetic field oscillations in Saturn's magnetosphere: Postequinox abrupt nonmonotonic transitions to northern system dominance, J. Geophys. Res., 118, 3243-3264, doi:10.1002/jgra.50186, 2013.

Ramer, K., Kivelson, M., Khurana, K., Sergis, N., Walker, R., and Jia, X.: Forces and Phases: An Investigation of Azimuthal Plasma and Field Periodicities in Saturn's Inner Magnetosphere, in: AGU Fall Meeting Abstracts, SM51B-2303, San Francisco, CA, USA, 3-7 December, 2012.

Southwood, D. J. and Kivelson, M. G.: Saturnian magnetospheric dynamics: Elucidation of a camshaft model, J. Geophys. Res., 112, 12222, doi:10.1029/2007JA012254, 2007.

Southwood, D. J. and Kivelson, M. G.: The source of Saturn's periodic radio emission, J. Geophys. Res., 114, 9201, doi:10.1029/2008JA013800, 2009.

Szego, K., Nemeth, Z., Erdos, G., Foldy, L., Thomsen, M., and Delapp, D.: The plasma environment of Titan: The magnetodisk of Saturn near the encounters as derived from ion densities measured by the Cassini/CAPS plasma spectrometer, J. Geophys. Res., 116, 10219, doi:10.1029/2011JA016629, 2011.

Szego, K., Nemeth, Z., Erdos, G., Foldy, L., Bebesi, Z., Thomsen, M., and Delapp, D.: Location of the magnetodisk in the nightside outer magnetosphere of Saturn near equinox based on ion densities, J. Geophys. Res., 117, 9225, doi:10.1029/2012JA017817, 2012.

Szego, K., Nemeth, Z., Foldy, L., Cowley, S. W. H., and Provan, G.: Dual periodicities in the flapping of Saturn's magnetodisk, J. Geophys. Res., 118, 2883-2887, doi:10.1002/jgra.50316, 2013.

Thomsen, M. F., Reisenfeld, D. B., Delapp, D. M., Tokar, R. L., Young, D. T., Crary, F. J., Sittler, E. C., McGraw, M. A., and Williams, J. D.: Survey of ion plasma parameters in Saturn's magnetosphere, J. Geophys. Res., 115, 10220 , doi:10.1029/2010JA015267, 2010.

Thomsen, M. F., Jackman, C. M., Tokar, R. L., and Wilson, R. J.: Plasma flows in Saturn's nightside magnetosphere, J. Geophys. Res., 119, 4521-4535, doi:10.1002/2014JA019912, 2014.

Wilson, R. J., Bagenal, F., Delamere, P. A., Desroche, M., Fleshman, B. L., and Dols, V.: Evidence from radial velocity measurements of a global electric field in Saturn's inner magnetosphere, J. Geophys. Res., 118, 2122-2132, doi:10.1002/jgra.50251, 2013.

Young, D. T., Berthelier, J. J., Blanc, M., Burch, J. L., Coates, A. J., Goldstein, R., Grande, M., Hill, T. W., Johnson, R. E., Kelha, V., McComas, D. J., Sittler, E. C., Svenes, K. R., Szego, K., Tanskanen, P., Ahola, K., Anderson, D., Bakshi, S., Baragiola, R. A., Barraclough, B. L., Black, R. K., Bolton, S., Booker, T., Bowman, R., Casey, P., Crary, F. J., Delapp, D., Dirks, G., Eaker, N., Funsten, H., Furman, J. D., Gosling, J. T., Hannula, H., Holmlund, C., Huomo, H., Illiano, J. M., Jensen, P., Johnson, M. A., Linder, D. R., Luntama, T., Maurice, S., McCabe, K. P., Mursula, K., Narheim, B. T., Nordholt, J. E., Preece, A., Rudzki, J., Ruitberg, A., Smith, K., Szalai, S., Thomsen, M. F., Viherkanto, K., Vilppola, J., Vollmer, T., Wahl, T. E., Wüest, M., Ylikorpi, T., and Zinsmeyer, C.: Cassini Plasma Spectrometer Investigation, Space Sci. Rev., 114, 1-112, doi:10.1007/s11214-004-1406-4, 2004. 